\title{
Caracterización de frutos de cinco genotipos de tacaco [Sechium tacaco (Pittier) C. Jeffrey] en Costa Rica
}

Fruit characterization of five genotypes of tacaco [Sechium tacaco (Pittier) C. Jeffrey] in Costa Rica

José Eladio Monge-Pérez¹, Michelle Loría-Coto

Fecha de recepción: 16 de noviembre de 2016

Fecha de aprobación: 8 de febrero de 2017

Monge-Pérez, J; Loría-Coto, M. Caracterización de frutos de cinco genotipos de tacaco [Sechium tacaco (Pittier) C. Jeffrey] en Costa Rica Tecnología en Marcha. Vol. 30-3. JulioSetiembre 2017. Pág 71-84.

DOI: $10.18845 /$ tm.v30i3.3274

1 Costarricense, ingeniero agrónomo. Estación Experimental Agrícola Fabio Baudrit Moreno y Sede de Guanacaste, Universidad de Costa Rica, Costa Rica. Correo electrónico: melonescr@yahoo.com.mx.

2 Costarricense, economista agrícola. Escuela de Ciencias Exactas y Naturales, Universidad Estatal a Distancia, Costa Rica. Correo electrónico: michelle_loria@yahoo.com. 


\title{
Palabras clave
}

Tacaco; Sechium tacaco; genotipos; peso del fruto; número de espinas; sabor; suturas longitudinales del fruto.

\section{Resumen}

Se caracterizaron los frutos de cinco genotipos de tacaco de alta calidad en Costa Rica, tanto a nivel cualitativo (tres variables) como cuantitativo (10 variables). Se presentaron diferencias estadísticamente significativas entre genotipos en el caso de las variables cuantitativas. Los datos muestran una amplia variabilidad en cuanto al peso del fruto $(31,55$ - 40,30 g), longitud del fruto $(57,36$ - 63,61 mm), ancho del fruto $(39,72-41,57 \mathrm{~mm})$, grosor del fruto $(28,01-30,40$ $\mathrm{mm})$, número de suturas longitudinales completas del fruto $(5,11-5,61)$, número de suturas longitudinales incompletas del fruto $(0,22-1,61)$, número de espinas del fruto $(0-22,36)$, relación largo/ancho del fruto $(1,42-1,53)$, relación largo/grosor del fruto $(1,92-2,21)$, y relación ancho/grosor del fruto $(1,36-1,45)$. Estos genotipos pueden ser buenas opciones para los productores y los consumidores que demandan frutos de tacaco de alta calidad. En la presente investigación se informa por primera vez sobre la existencia de frutos de tacaco con seis y siete suturas longitudinales completas, y con entre dos y cinco suturas longitudinales incompletas, lo que representa un hallazgo que puede ilustrar los cambios evolutivos recientes de esta especie.

\section{Keywords}

Tacaco; Sechium tacaco; genotypes; fruit weight; number of spines; taste; fruit longitudinal sutures.

\begin{abstract}
Tacaco fruits of five high quality genotypes were characterized in Costa Rica, both at qualitative (three variables) and quantitative (10 variables) levels. There were statistically significant differences between genotypes for quantitative variables. Data show a wide variability with respect to fruit weight $(31,55-40,30 \mathrm{~g})$, fruit length $(57,36-63,61 \mathrm{~mm})$, fruit width $(39,72-$ $41,57 \mathrm{~mm})$, fruit thickness $(28,01-30,40 \mathrm{~mm})$, number of complete fruit longitudinal sutures $(5,11-5,61)$, number of incomplete fruit longitudinal sutures $(0,22-1,61)$, number of fruit spines $(0-22,36)$, fruit length/width ratio $(1,42-1,53)$, fruit length/thickness ratio $(1,92-2,21)$, and fruit width/thickness ratio $(1,36-1,45)$. These genotypes could be good choices for growers and consumers that demand high quality tacaco fruits. This is the first notice about the existence of tacaco fruits with six and seven complete longitudinal sutures, and with two to five incomplete longitudinal sutures; this could illustrate the recent evolutive changes of this species.
\end{abstract}

\section{Introducción}

El tacaco es una especie vegetal que presenta una planta herbácea, trepadora, anual y monoica; pertenece a la familia Cucurbitaceae y es de interés alimenticio. Su nombre científico actual es [Sechium tacaco (Pittier) C. Jeffrey], aunque inicialmente se le nombró como Polakowskia tacaco por Pittier en 1910, y luego fue cambiado a Frantzia tacaco por Wunderlin en 1976 [1], [2]. 
El centro de origen del tacaco es Costa Rica, pues además de que es en este país donde crece su pariente silvestre más cercano, $S$. talamancensis, es también el único país en donde se sabe que la especie es y ha sido cultivada; es decir, que la distribución geográfica del tacaco se circunscribe únicamente al territorio costarricense [2], [3]. El tacaco es una de las dos únicas especies endémicas de Costa Rica, junto con la cabuya, Furcraea cabuya [4]. Por lo tanto, a juicio de los autores, el tacaco debe considerarse un patrimonio natural de Costa Rica.

Entre el año 2001 y el 2010, la cantidad de tacaco comercializado en el CENADA (principal mercado mayorista de hortalizas de Costa Rica) varió entre 32160 y 66050 kg por año; el valor total del tacaco comercializado en ese período osciló entre 8,8 y 36,2 millones de colones por año; la procedencia de ese producto durante todos los años mencionados correspondió en un 100 \% al cantón de Paraíso, en Cartago. La oferta de tacaco se presentó en todos los meses del año para todos los años; el precio promedio osciló entre 275 colones/kg en el 2002, y 1080 colones/kg en el 2009 [5].

El cultivo del tacaco se practica preferentemente en zonas con altitudes entre 1000 y 1700 msnm, y es posible realizarlo prácticamente en cualquier época del año [2]. Sin embargo, también se han observado plantas de tacaco con excelentes producciones en Alajuela centro (914 msnm) (J. E. Monge-Pérez, datos sin publicar) y Puente de Piedra de Grecia (800 msnm aproximadamente) [6]. En 1992 se tenía información sobre la presencia de tacaco únicamente en el Valle Central (Oriental y Occidental) y en la región Brunca (Pérez Zeledón y Coto Brus) de Costa Rica [1]. Sin embargo, recientemente se conoce también sobre su presencia en la zona alta de la Cordillera de Tilarán, en Cedral de Montes de Oro, a unos 800-1000 msnm [7], así como en Monteverde, Puntarenas, y en las zonas altas del cantón de Tilarán, Guanacaste (J. E. Monge-Pérez, datos sin publicar), y también en la zona montañosa de la Península de Nicoya, en Quebrada Grande de Nandayure, Guanacaste, aproximadamente a unos 500 msnm [8]. Asimismo, hay evidencia de que algunos costarricenses han llevado el cultivo a la zona de Volcán, provincia de Chiriquí, Panamá (J. E. Monge-Pérez, datos sin publicar).

Los frutos de tacaco se consumen ya sea como verdura cocida en sopas, en picadillo, en encurtidos y en miel, simplemente hervidos como bocadillo, y principalmente son muy apreciados como parte fundamental del guiso tradicional costarricense llamado "olla de carne" [1], [2]. Las hojas se usan en la elaboración de albóndigas y tortas a base de carne molida. Del fruto tierno se consumen todas las partes, incluyendo la cáscara y la semilla, mientras que del fruto maduro estas últimas son removidas [9]. El fruto tierno se puede consumir "picado" con arroz, en "arroz guacho", en sopas, y como minivegetal en ensaladas [10]. El tacaco supera en contenido proteico y de fósforo a otras cucurbitáceas como chayote, pepino, melón, sandía y zucchini [1], [9].

El tacaco es un cultivo de huerto, solar o traspatio, de tal forma que sus plantaciones son pequeñas y la producción está destinada principalmente al autoconsumo, y sólo en algunos casos en los que se logran generar ciertos excedentes, estos son comercializados en los mercados [2], lo cual sucede principalmente en la zona de La Flor y El Yas de Paraíso, Cartago, Costa Rica (J. E. Monge-Pérez, datos sin publicar).

La época de mayor producción de tacaco en Costa Rica comprende desde agosto hasta diciembre, aunque se puede prolongar hasta marzo [2].

En los frutos sazones, el epicarpo (cáscara) es duro y se remueve después del proceso de cocción, y el mesocarpo está constituido de tejido parenquimatoso cargado de gránulos de almidón, y presenta en algunos tipos una red de fibras muy desarrolladas (Ilamada "estopa") que acompañan a los elementos vasculares y que lo atraviesan en distintas direcciones, lo que reduce su calidad culinaria. Las semillas maduras presentan sabor amargo por lo que debe evitarse su consumo [1], [11]. 
Según diversos investigadores, el tamaño del fruto varía entre 4,0 y 7,0 cm de largo, entre 3,0 y 4,5 cm de ancho, y entre 1,5 y 3,0 cm de grosor; el peso de los frutos varía entre 22,7 y 42,2 g; y en los genotipos con espinas, la cantidad de espinas por fruto varía entre 16 y 26 [12], [1], [13], [11], [14], [15], [16], [10]. Además, diversos autores mencionan que el fruto presenta únicamente cinco suturas longitudinales (o surcos) [12], [3], [13], [11], [2].

Se ha informado de una tendencia de reducción en la longitud, ancho, grosor y peso de los frutos conforme transcurren las semanas de producción; además, la cantidad de espinas es menor al inicio de la cosecha y tiende al aumento hasta la octava semana de cosecha, después de la cual decrece ligeramente [16].

No se conocen variedades comerciales de tacaco [17]. El estado semisilvestre de su domesticación y la limitada importancia económica de la planta ha sido obstáculo para el impulso de investigaciones agronómicas y fitogenéticas. Además, la carencia de recomendaciones técnicas y de variedades superiores ocasiona que los agricultores potenciales de esta especie no se arriesguen a producir [18]. El tacaco se cultiva generalmente como un componente de los huertos mixtos, y existe poca investigación sobre su manejo agronómico con fines comerciales [15].

Según un investigador, algunas plantas tienden a producir frutos sin espinas, y estos suelen estar asociados con un bajo contenido de fibra en el mesocarpo ("estopa") [11]. Otro autor informó que existen cuatro tipologías básicas del fruto: con espinas y con estopa, con espinas y sin estopa, sin espinas y con estopa, y sin espinas y sin estopa [12]. También se conoce la existencia de variabilidad en cuanto a otros caracteres del fruto como tamaño, distribución de las espinas y sabor [1].

Algunos productores de tacaco consideran que las espinas dificultan los procesos de cosecha, empaque y limpieza del fruto, por lo que prefieren materiales con pocas espinas o sin ellas, pero otros agricultores prefieren los tacacos con espinas, debido a que consideran que tienen una mayor productividad [10]. Sin embargo, hasta el momento no se han realizado investigaciones sobre la relación entre presencia o ausencia de espinas, y la productividad del tacaco.

Desde 1991, se consideraba que el tacaco, el único aporte de Costa Rica a la cultura agrícola mundial, estaba a punto de desaparecer ante la imposición de prácticas productivas que privilegiaban los cultivos exportables y los cambios en los hábitos de consumo [19]. En ese momento esta hortaliza se conseguía esporádicamente en los mercados locales y su cultivo solía estar ligado a las áreas de pobreza, pues su cotización en los mismos era baja, y fuera del país era prácticamente desconocido. Por lo tanto, su importancia económica era virtualmente nula. También se consideraba que el tacaco en Costa Rica era una víctima de los cambios productivos y que podría desaparecer como producto alimenticio disponible en el país [19]. A juicio de los autores, esta amenaza sigue estando presente hoy en día.

En 1992 ya se consideraba la posibilidad de que se estuviera perdiendo variabilidad genética en tacaco, debido al efecto de algunos factores como el desconocimiento sobre la existencia de recursos vegetales originarios del país, la destrucción de bosques, el menosprecio a los cultivos autóctonos, y la falta de tecnología en el cultivo y utilización de esta planta. En ese momento se identificaron los siguientes obstáculos existentes para la promoción del cultivo del tacaco: actitud cultural de menosprecio a los recursos autóctonos (cambio cultural), la dificultad en la propagación de la especie, el desconocimiento sobre la existencia de variedades de reconocida calidad superior, y la falta de investigación en cuanto a aspectos agronómicos [1].

El objetivo de esta investigación fue caracterizar los frutos de cinco genotipos de tacaco con buenas características de calidad, colectados en diversas regiones de Costa Rica. 


\section{Materiales y métodos}

En una primera etapa se visitaron varias zonas de Costa Rica entre junio y julio de 2013, con el fin de identificar diferentes genotipos de tacaco [Sechium tacaco (Pittier) C. Jeffrey]. Los cantones o sitios visitados fueron: Tilarán, Puntarenas, Abangares, Liberia, Bagaces, Nicoya, Santa Cruz, Cañas, Monteverde, San Carlos, Zarcero, San Ramón, Palmares, Naranjo, Valverde Vega, Poás, Grecia, Alajuela, San Carlos, Cartago, Paraíso, Turrialba, Dota, Tarrazú, Pérez Zeledón y Coto Brus.

Como conclusión de la primera etapa, se reconocieron nueve genotipos, de los cuales se seleccionaron los cinco que presentaron las mejores características de calidad, especialmente buen sabor, y poca o nula presencia de fibras en el mesocarpo. Posteriormente, se realizó la colecta de frutos de esos cinco genotipos; se recolectaron entre 28 y 55 frutos sazones por genotipo. Las colectas se realizaron entre los meses de junio de 2013 y octubre de 2015, en los sitios descritos en el cuadro 1.

Se evaluaron las siguientes variables:

\section{A. Cuantitativas:}

1. Peso del fruto (g): se evaluó el peso de cada uno de los frutos colectados, y se obtuvo el promedio. Se utilizó una balanza electrónica marca Salter Brecknell, modelo PB500, con una capacidad de 500,0 \pm 0,1 g.

2. Longitud del fruto ( $\mathrm{mm}$ ): se midió la longitud de cada uno de los frutos colectados, y se obtuvo el promedio. Para esta variable, así como para el ancho y grosor del fruto, se utilizó un calibrador electrónico marca Pittsburgh con una capacidad de 154,5 0 0,1 mm.

3. Ancho del fruto $(\mathrm{mm})$ : se evaluó el ancho de cada uno de los frutos colectados, y se obtuvo el promedio.

4. Grosor del fruto (mm): se midió el grosor de cada uno de los frutos colectados, y se obtuvo el promedio.

5. Número de suturas longitudinales completas: se registró el número de suturas longitudinales completas de cada uno de los frutos colectados, y se obtuvo el promedio.

6. Número de suturas longitudinales incompletas: se registró el número de suturas longitudinales incompletas (que no llegan hasta alguno de los extremos del fruto) de cada uno de los frutos colectados, y se obtuvo el promedio.

7. Número de espinas: se registró el número total de espinas presentes en la cáscara de cada uno de los frutos colectados, y se obtuvo el promedio.

8. Relación largo/ancho del fruto: se obtuvo al dividir la longitud (en $\mathrm{mm}$ ) entre el ancho (en $\mathrm{mm}$ ) para cada uno de los frutos, y se obtuvo el promedio.

9. Relación largo/grosor del fruto: se obtuvo al dividir la longitud (en mm) entre el grosor (en $\mathrm{mm}$ ) para cada uno de los frutos, y se obtuvo el promedio.

10. Relación ancho/grosor del fruto: se obtuvo al dividir el ancho (en mm) entre el grosor (en $\mathrm{mm}$ ) para cada uno de los frutos, y se obtuvo el promedio.

\section{B. Cualitativas:}

Luego de evaluar las variables cuantitativas, se procedió a colocar 10 frutos de tacaco de cada genotipo en agua hirviendo durante 15 minutos, y luego se les quitó la cáscara y se separó la semilla, y con el mesocarpo se procedió a evaluar las siguientes características: 
1. Presencia de fibras en el mesocarpo: Se evaluó la presencia o ausencia de fibras en el mesocarpo del fruto, al momento de consumirlo.

2. Consistencia del mesocarpo: Se evaluó la consistencia del mesocarpo del fruto, al momento de consumirlo.

3. Sabor del fruto: Se evaluó el sabor mediante una prueba de degustación (también llamada análisis sensorial), llevada a cabo por diez personas consumidoras de tacaco.

Para cada una de las variables cuantitativas se realizó una comparación entre las medias obtenidas por cada genotipo, por medio de una prueba de t de Student con una significancia de $5 \%$, para descartar o confirmar diferencias significativas entre ellos.

Cuadro 1. Sitios de recolección de los cinco genotipos de tacaco seleccionados.

\begin{tabular}{|c|c|c|c|c|c|}
\hline \multirow{2}{*}{ Identificación } & \multirow{2}{*}{$\begin{array}{l}\text { Lugar de } \\
\text { recolección }\end{array}$} & \multirow{2}{*}{$\begin{array}{l}\text { Número } \\
\text { de frutos } \\
\text { evaluados }\end{array}$} & \multicolumn{2}{|c|}{ Coordenadas geográficas } & \multirow{2}{*}{$\begin{array}{l}\text { Altitud } \\
\text { (msnm) }\end{array}$} \\
\hline & & & Latitud Norte & Longitud Oeste & \\
\hline 1 & Escazú, San José & 34 & $9^{\circ} 54^{\prime} 55,545^{\prime \prime}$ & $84^{\circ} 8^{\prime} 3,581^{\prime \prime}$ & 1147 \\
\hline 2 & $\begin{array}{c}\text { El Yas de Paraíso, } \\
\text { Cartago }\end{array}$ & 28 & $9^{\circ} 51^{\prime} 41,433 \prime$ & $83^{\circ} 49^{\prime} 12,375^{\prime \prime}$ & 1261 \\
\hline 3 & $\begin{array}{c}\text { La Flor de Paraíso, } \\
\text { Cartago }\end{array}$ & 28 & $9^{\circ} 52^{\prime} 13,048 "$ & $83^{\circ} 48^{\prime} 28,142^{\prime \prime}$ & 1264 \\
\hline 7 & San Isidro de Alajuela & 55 & $10^{\circ} 4^{\prime} 49,061^{\prime \prime}$ & $84^{\circ} 11^{\prime} 43,073^{\prime \prime}$ & 1363 \\
\hline 9 & $\begin{array}{c}\text { El Dos de Tilarán, } \\
\text { Guanacaste }\end{array}$ & 54 & $10^{\circ} 22^{\prime} 41,174 "$ & $84^{\circ} 53^{\prime} 57,627^{\prime \prime}$ & 939 \\
\hline
\end{tabular}

\section{Resultados y discusión}

En el cuadro 2 se presentan los datos para las variables cualitativas evaluadas. A pesar de que los cinco genotipos presentan un sabor aceptable, el genotipo 3 presentó una consistencia "hulosa" (o más firme) del mesocarpo, lo cual resulta menos agradable al paladar de los consumidores, en relación a la consistencia "harinosa" (o más suave y esponjosa). En la figura 1 se muestran los frutos de tres de los genotipos evaluados.

Cuadro 2. Características cualitativas de los frutos de los genotipos de tacaco.

\begin{tabular}{|c|c|c|c|}
\hline Genotipo & Presencia de fibras en el mesocarpo & $\begin{array}{c}\text { Consistencia del } \\
\text { mesocarpo }\end{array}$ & Sabor \\
\hline 1 & Ninguna o muy poca & "Harinosa" & Muy bueno \\
\hline 2 & Algo de fibra & "Harinosa" & Aceptable \\
\hline 3 & Poca & "Hulosa" & Aceptable \\
\hline 7 & Ninguna o muy poca & "Harinosa", seca & Bueno \\
\hline
\end{tabular}




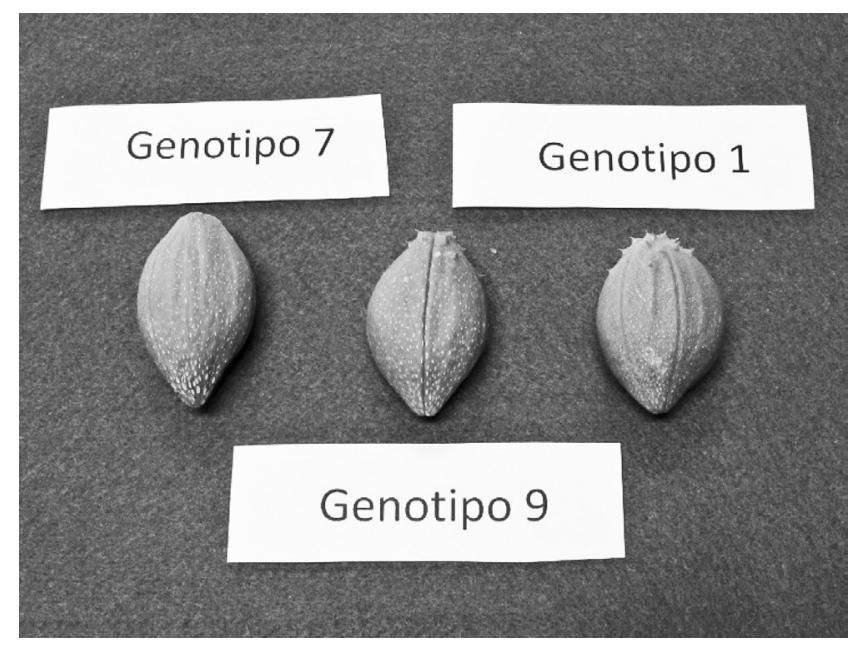

Figura 1. Frutos de tacaco de tres de los genotipos evaluados.

En el cuadro 3 se muestran los datos para el peso del fruto. Se presentan diferencias significativas entre genotipos para esta variable; el genotipo 1 es el que presenta los frutos con menor peso, y el genotipo 7 presenta los frutos con mayor peso. Los datos obtenidos por los genotipos 3 y 7 se encuentran por encima del rango de peso del fruto hallado por otros investigadores para esta característica, de entre 22,7 y 42,2 g [1], [14], [15], [16], [10], ya que presentaron algunos frutos con un peso de hasta 48,9 y 49,0 g; los valores obtenidos por los otros tres genotipos sí se ubicaron dentro del rango mencionado. Todos los cinco genotipos presentaron un peso promedio del fruto superior al informado por un investigador, de 27,2 g [13], y al informado por otro autor, de 29,6 g [14], para el tacaco.

Cuadro 3. Peso del fruto de los genotipos de tacaco.

\begin{tabular}{|c|c|c|c|c|}
\hline \multirow{2}{*}{ Genotipo } & \multicolumn{4}{|c|}{ Peso del fruto (g) } \\
\hline 1 & Promedio & Rango & Mediana & $\begin{array}{c}\text { Coeficiente de } \\
\text { variación (\%) }\end{array}$ \\
\hline 9 & $31,55 \mathrm{a}$ & $25,9-39,3$ & 31,15 & 12,28 \\
\hline 2 & $34,04 \mathrm{~b}$ & $25,7-41,4$ & 33,80 & 10,73 \\
\hline 3 & $35,44 \mathrm{bc}$ & $28,8-41,2$ & 35,15 & 10,17 \\
\hline 7 & $36,71 \mathrm{c}$ & $29,5-49,0$ & 36,30 & 12,71 \\
\hline
\end{tabular}

Nota: Letras distintas indican diferencias significativas ( $p \leq 0,05)$, según la prueba de t de Student.

En el cuadro 4 se presentan los datos para la longitud del fruto. El genotipo 1 presenta los frutos con menor longitud, y el genotipo 7 los de mayor longitud; esto se relaciona directamente con el peso de los frutos. Estos datos se ubican dentro del rango de longitud del fruto informado por otros investigadores, de entre 40 y $70 \mathrm{~mm}$ [3], [11], [10], [12]. Sin embargo, ninguno de los genotipos estudiados en este trabajo alcanzó los valores de longitud de fruto encontrados en otro genotipo colectado en Alajuela centro (identificado como JM-2); al evaluar cinco frutos de 
dicho genotipo se obtuvo una longitud promedio de 69,58 mm, con un mínimo de 65,4 mm y un máximo de $72,5 \mathrm{~mm}$, aunque otros frutos mostraron casi $80 \mathrm{~mm}$ de longitud (figura 2); ese genotipo no se evaluó en esta investigación debido a la presencia de fibras en el mesocarpo (J. E. Monge-Pérez, datos sin publicar).

Cuadro 4. Longitud del fruto de los genotipos de tacaco.

\begin{tabular}{|c|c|c|c|c|}
\hline \multirow{2}{*}{ Genotipo } & \multicolumn{4}{|c|}{ Longitud del fruto $(\mathrm{mm})$} \\
\hline 1 & Promedio & Rango & Mediana & $\begin{array}{c}\text { Coeficiente de } \\
\text { variación (\%) }\end{array}$ \\
\hline 2 & $57,36 \mathrm{a}$ & $52,6-64,0$ & 57,25 & 5,14 \\
\hline 3 & $57,45 \mathrm{a}$ & $53,7-62,4$ & 57,10 & 4,66 \\
\hline 9 & $59,03 \mathrm{~b}$ & $54,5-67,0$ & 58,25 & 5,40 \\
\hline 7 & $61,56 \mathrm{c}$ & $54,4-68,1$ & 61,75 & 4,36 \\
\hline & $63,61 \mathrm{~d}$ & $56,6-67,7$ & 64,00 & 3,32 \\
\hline
\end{tabular}

Nota: Letras distintas indican diferencias significativas $(p \leq 0,05)$, según la prueba de t de Student.

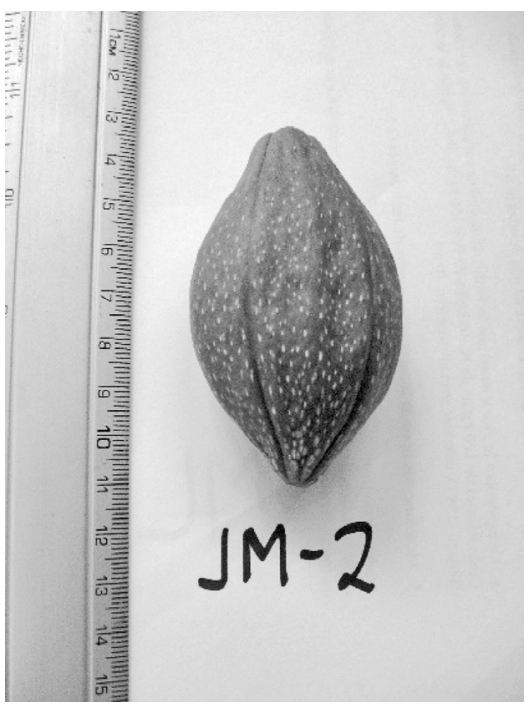

Figura 2. Genotipo de tacaco colectado en Alajuela centro.

En el cuadro 5 se muestran los datos para el ancho del fruto; nuevamente el genotipo 1 muestra los valores más bajos, y el genotipo 7 los más altos, lo que de igual manera se relaciona directamente con el peso de los frutos. Los datos obtenidos coinciden con el rango de ancho del fruto hallado por otro autor, de entre 30 y $45 \mathrm{~mm}$ [11], [10], [12].

En el cuadro 6 se presentan los datos para el grosor del fruto. En este caso, el genotipo con los frutos con menor grosor fue el genotipo 9, y el genotipo 7 presentó los frutos con el mayor grosor. Estos datos se ubican por encima del rango de grosor del fruto obtenido por un investigador, de entre 20 y $30 \mathrm{~mm}$ [11], y por otro autor, de entre 15 y $25 \mathrm{~mm}$ [12], pues en todos los genotipos se encontraron frutos con más de $30 \mathrm{~mm}$ de grosor. 
Cuadro 5. Ancho del fruto de los genotipos de tacaco.

\begin{tabular}{|c|c|c|c|c|}
\hline & \multicolumn{4}{|c|}{ Ancho del fruto $(\mathrm{mm})$} \\
\hline Genotipo & Promedio & Rango & Mediana & Coeficiente de variación (\%) \\
\hline 1 & $39,72 \mathrm{a}$ & $36,6-43,9$ & 39,60 & 4,81 \\
\hline 9 & $40,36 \mathrm{a}$ & $35,9-43,8$ & 40,45 & 4,70 \\
\hline 2 & $40,59 \mathrm{ab}$ & $37,2-43,8$ & 40,45 & 3,96 \\
\hline 3 & $41,43 \mathrm{bc}$ & $37,8-44,9$ & 41,75 & 4,80 \\
\hline 7 & $41,57 \mathrm{c}$ & $39,2-44,5$ & 41,60 & 2,72 \\
\hline
\end{tabular}

Nota: Letras distintas indican diferencias significativas $(p \leq 0,05)$, según la prueba de te Student.

Cuadro 6. Grosor del fruto de los genotipos de tacaco.

\begin{tabular}{|c|c|c|c|c|}
\hline & \multicolumn{4}{|c|}{ Grosor del fruto $(\mathrm{mm})$} \\
\hline Genotipo & Promedio & Rango & Mediana & Coeficiente de variación (\%) \\
\hline 9 & $28,01 \mathrm{a}$ & $24,1-32,5$ & 28,20 & 6,56 \\
\hline 1 & $28,54 \mathrm{a}$ & $25,8-31,1$ & 28,65 & 4,22 \\
\hline 3 & $29,61 \mathrm{~b}$ & $27,3-32,3$ & 29,30 & 4,81 \\
\hline 2 & $30,00 \mathrm{bc}$ & $27,2-35,1$ & 29,95 & 5,60 \\
\hline 7 & $30,40 \mathrm{c}$ & $28,1-32,6$ & 30,40 & 3,25 \\
\hline
\end{tabular}

Nota: Letras distintas indican diferencias significativas $(p \leq 0,05)$, según la prueba de t de Student.

En el cuadro 7 se muestran los resultados obtenidos para el número de suturas longitudinales completas del fruto. Para esta característica, el genotipo 9 presentó los frutos con el menor número de suturas longitudinales completas, y el genotipo 2 mostró los frutos con el mayor número. Las investigaciones previas habían establecido que el fruto de tacaco tiene únicamente cinco suturas longitudinales [12], [3], [13], [11], [2]. Sin embargo, en la presente investigación se demostró que en los cinco genotipos se presentaron algunos frutos con seis y hasta siete suturas longitudinales completas, lo que constituye un hallazgo de la presente investigación (figuras 3 y 4 ).

Cuadro 7. Número de suturas longitudinales completas del fruto de los genotipos de tacaco.

\begin{tabular}{|c|c|c|c|c|}
\hline & \multicolumn{4}{|c|}{ Número de suturas longitudinales completas del fruto } \\
\hline Genotipo & Promedio & Rango & Mediana & Coeficiente de variación (\%) \\
\hline 9 & $5,11 \mathrm{a}$ & $5-6$ & 5 & 6,21 \\
\hline 3 & $5,14 \mathrm{ab}$ & $5-6$ & 5 & 6,93 \\
\hline 1 & $5,35 \mathrm{bc}$ & $5-6$ & 5 & 9,06 \\
\hline 7 & $5,42 \mathrm{c}$ & $5-7$ & 5 & 9,85 \\
\hline 2 & $5,61 \mathrm{c}$ & $5-7$ & 6 & 11,22 \\
\hline
\end{tabular}

Nota: Letras distintas indican diferencias significativas $(p \leq 0,05)$, según la prueba de t de Student. 


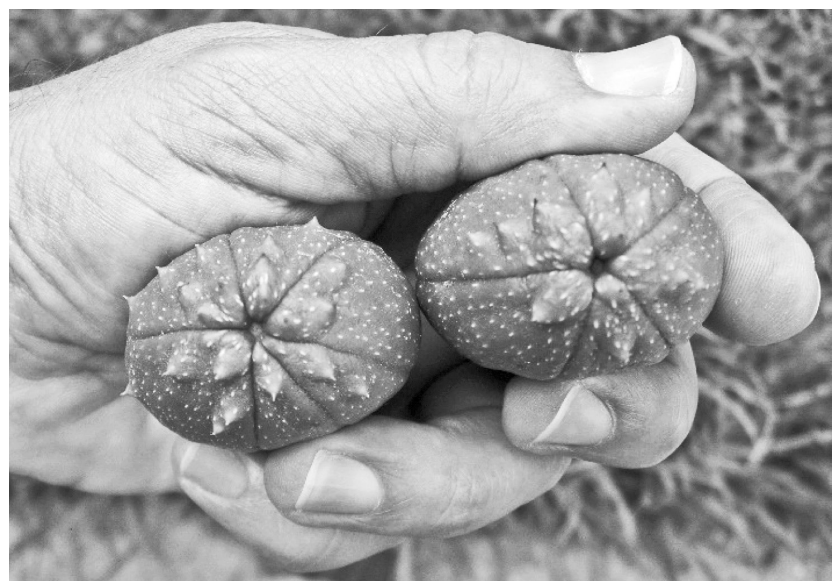

Figura 3. Frutos de tacaco con seis y cinco suturas longitudinales completas, respectivamente.

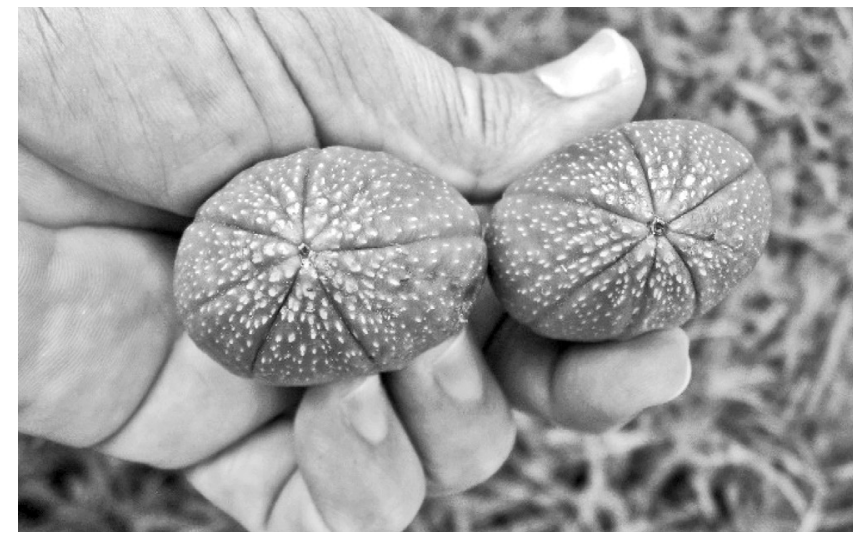

Figura 4. Frutos de tacaco con siete suturas longitudinales completas.

En el cuadro 8 se presentan los datos para el número de suturas longitudinales incompletas del fruto. En este caso, también fue el genotipo 9 el que mostró los frutos con menor cantidad de suturas longitudinales incompletas, y el genotipo 3 presentó los frutos con la mayor cantidad. Se debe resaltar que esta variable presentó un coeficiente de variación muy alto, en comparación a las otras variables. Ningún investigador había informado anteriormente sobre la existencia de suturas longitudinales incompletas en los frutos de tacaco (es decir, que no llegan hasta alguno de los extremos del fruto), lo que también constituye un hallazgo para esta especie en la presente investigación (figura 5). Para todos los cinco genotipos se encontraron algunos frutos con entre dos y cinco suturas longitudinales incompletas.

En el cuadro 9 se muestran los resultados obtenidos para el número de espinas en el fruto. Los genotipos 3 y 7 presentaron frutos sin espinas o casi sin ellas (unos pocos frutos del genotipo 7 presentaban una o dos espinas), mientras que los demás genotipos sí mostraron muchas espinas. En este caso, también el coeficiente de variación obtenido fue alto. Diversos investigadores han encontrado en los genotipos de tacaco con espinas, que la cantidad de espinas por fruto varía entre 16 y 26 [1], [14], [15], [16]. Por otra parte, otro autor encontró que el número promedio de espinas por fruto fue de 21,52, con un mínimo de nueve y un máximo de 36 [13]. Sin embargo, en el presente trabajo se encontró un rango más amplio para esta variable en los genotipos con espinas, con un mínimo de siete y un máximo de 40 espinas por fruto. 
Cuadro 8. Número de suturas longitudinales incompletas del fruto de los genotipos de tacaco.

\begin{tabular}{|c|c|c|c|c|}
\hline & \multicolumn{4}{|c|}{ Número de suturas longitudinales incompletas del fruto } \\
\hline Genotipo & Promedio & Rango & Mediana & Coeficiente de variación (\%) \\
\hline 9 & $0,22 \mathrm{a}$ & $0-2$ & 0 & 208,09 \\
\hline 7 & $0,47 \mathrm{~b}$ & $0-2$ & 0 & 127,8 \\
\hline 1 & $0,74 \mathrm{~b}$ & $0-4$ & 0 & 139,29 \\
\hline 2 & $1,29 \mathrm{c}$ & $0-3$ & 1 & 78,80 \\
\hline 3 & $1,61 \mathrm{c}$ & $0-5$ & 1,5 & 70,52 \\
\hline
\end{tabular}

Nota: Letras distintas indican diferencias significativas $(p \leq 0,05)$, según la prueba de t de Student.

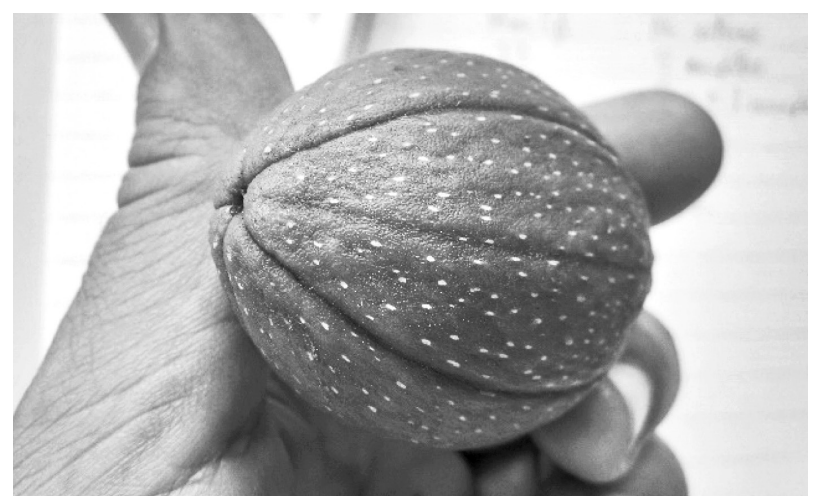

Figura 5. Fruto de tacaco con una sutura longitudinal incompleta en primer plano.

Cuadro 9. Número de espinas del fruto de los genotipos de tacaco.

\begin{tabular}{|c|c|c|c|c|}
\hline & \multicolumn{4}{|c|}{ Número de espinas del fruto } \\
\hline Genotipo & Promedio & Rango & Mediana & Coeficiente de variación (\%) \\
\hline 3 & $0,00 \mathrm{a}$ & 0 & 0 & nd \\
\hline 7 & $0,13 \mathrm{a}$ & $0-2$ & 0 & 339,96 \\
\hline 9 & $18,83 \mathrm{~b}$ & $7-28$ & 19 & 21,58 \\
\hline 1 & $22,09 \mathrm{c}$ & $14-32$ & 22 & 23,12 \\
\hline 2 & $22,36 \mathrm{c}$ & $10-40$ & 23 & 38,9 \\
\hline
\end{tabular}

Nota: Letras distintas indican diferencias significativas $(p \leq 0,05)$, según la prueba de t de Student.

En el cuadro 10 se presentan los datos para la relación largo/ancho del fruto. Los frutos con una mayor relación largo/ancho (forma más "alargada") fueron los de los genotipos 9 y 7 . Sin embargo, ninguno de los genotipos obtuvo una relación largo/ancho tan amplia como la encontrada en otro genotipo colectado en Alajuela centro, con un valor de 1,69 (J. E. MongePérez, datos sin publicar).

En el cuadro 11 se presentan los datos para la relación largo/grosor del fruto. El genotipo 2 presentó los frutos con la menor relación largo/grosor (más "gordos"), y el genotipo 9 los frutos 
con la mayor magnitud para esta característica (menos "gordos"). En este caso, igualmente ninguno de los genotipos estudiados alcanzó una relación largo/grosor tan amplia como la encontrada en otro genotipo colectado en Alajuela centro, con un valor de 2,38 (J. E. MongePérez, datos sin publicar).

Cuadro 10. Relación largo/ancho de los frutos de los genotipos de tacaco.

\begin{tabular}{|c|c|c|c|c|}
\hline & \multicolumn{4}{|c|}{ Relación largo/ancho de los frutos } \\
\hline Genotipo & Promedio & Rango & Mediana & Coeficiente de variación (\%) \\
\hline 2 & $1,42 \mathrm{a}$ & $1,32-1,52$ & 1,41 & 4,08 \\
\hline 3 & $1,43 \mathrm{a}$ & $1,32-1,55$ & 1,43 & 4,02 \\
\hline 1 & $1,45 \mathrm{a}$ & $1,25-1,58$ & 1,44 & 4,33 \\
\hline 9 & $1,53 \mathrm{~b}$ & $1,39-1,65$ & 1,53 & 4,11 \\
\hline 7 & $1,53 \mathrm{~b}$ & $1,43-1,63$ & 1,53 & 3,02 \\
\hline
\end{tabular}

Nota: Letras distintas indican diferencias significativas $(p \leq 0,05)$, según la prueba de t de Student.

Cuadro 11. Relación largo/grosor de los frutos de los genotipos de tacaco.

\begin{tabular}{|c|c|c|c|c|}
\hline & \multicolumn{4}{|c|}{ Relación largo/grosor de los frutos } \\
\hline Genotipo & Promedio & Rango & Mediana & Coeficiente de variación (\%) \\
\hline 2 & $1,92 \mathrm{a}$ & $1,69-2,24$ & 1,91 & 5,65 \\
\hline 3 & $1,99 \mathrm{~b}$ & $1,82-2,24$ & 1,98 & 4,98 \\
\hline 1 & $2,01 \mathrm{~b}$ & $1,75-2,21$ & 2,02 & 5,34 \\
\hline 7 & $2,09 \mathrm{c}$ & $1,94-2,27$ & 2,09 & 3,56 \\
\hline 9 & $2,21 \mathrm{~d}$ & $1,74-2,53$ & 2,22 & 8,18 \\
\hline
\end{tabular}

Nota: Letras distintas indican diferencias significativas $(p \leq 0,05)$, según la prueba de t de Student.

En el cuadro 12 se presentan los datos para la relación ancho/grosor del fruto. En este caso, se repitió la tendencia presentada para la variable largo/grosor del fruto, donde el genotipo 2 presentó los frutos con la menor relación ancho/grosor (menos "achatados"), y el genotipo 9 con el mayor valor para esta variable (más "achatados").

Cuadro 12. Relación ancho/grosor de los frutos de los genotipos de tacaco.

\begin{tabular}{|c|c|c|c|c|}
\hline & \multicolumn{4}{|c|}{ Relación ancho/grosor de los frutos } \\
\hline Genotipo & Promedio & Rango & Mediana & Coeficiente de variación (\%) \\
\hline 2 & $1,36 \mathrm{a}$ & $1,14-1,50$ & 1,38 & 5,67 \\
\hline 7 & $1,37 \mathrm{a}$ & $1,29-1,43$ & 1,36 & 1,96 \\
\hline 1 & $1,39 \mathrm{~b}$ & $1,32-1,49$ & 1,39 & 3,40 \\
\hline 3 & $1,40 \mathrm{~b}$ & $1,31-1,47$ & 1,40 & 3,06 \\
\hline 9 & $1,45 \mathrm{c}$ & $1,17-1,65$ & 1,47 & 7,87 \\
\hline
\end{tabular}

Nota: Letras distintas indican diferencias significativas $(p \leq 0,05)$, según la prueba de te Student. 


\section{Conclusiones y recomendaciones}

Los genotipos seleccionados en esta investigación constituyen buenas opciones para los productores y los consumidores que demandan tacacos de alta calidad, debido a sus excelentes características organolépticas (buen sabor, y poca o nula presencia de fibras en el mesocarpo).

Este trabajo constituye la primera caracterización cuantitativa de diferentes genotipos de tacaco a nivel de fruto; se evidenciaron diferencias estadísticamente significativas entre los genotipos para las diversas variables evaluadas.

En la presente investigación se informa por primera vez sobre la existencia de frutos de tacaco con seis y siete suturas longitudinales completas, y con entre dos y cinco suturas longitudinales incompletas, lo que representa un hallazgo que puede ilustrar los cambios evolutivos recientes de esta especie.

\section{Agradecimientos}

Los autores agradecen el financiamiento recibido por parte de la Vicerrectoría de Investigación de la Universidad de Costa Rica, así como la colaboración de Cristina Arguedas, Eugenia Araya, Marielos Corella, Ingrid Arias, Vanessa Vargas, Sandra Arias, Magaly Guerrero, Ceidy Coto, Eladio Monge, Álvaro Loría, Freddy Chinchilla y Asdrúbal Rodríguez para la realización de este trabajo, así como de Mario Monge en la revisión de la traducción del resumen al inglés.

\section{Referencias}

[1] A. Brenes, «Situación actual y perspectivas del tacaco [Sechium tacaco (Pittier) C. Jeffrey] en Costa Rica,» Boletín Agrario, vol. 11, n 39, pp. 1-23, 1992.

[2] R. Lira-Saade, Estudios taxonómicos y ecogeográficos de las Cucurbitaceae latinoamericanas de importancia económica, Roma, Italia: International Plant Genetic Resources Institute, 1995.

[3] J. León, Botánica de los cultivos tropicales, San José, Costa Rica: IICA, 1987.

[4] Instituto Nacional de Biodiversidad, «Los ecosistemas agrícolas. Estudio de país: Costa Rica; estudio nacional de biodiversidad; 3.6 Especies domesticadas o cultivadas,» 2004. [En línea]. Available: http://www.inbio.ac.cr/ estrategia/Estudio_2004/Paginas/ecosistema03.html. [Último acceso: 2016].

[5] Programa Integral de Mercadeo Agropecuario, «Estadísticas sobre la comercialización de tacaco en el CENADA, Costa Rica,» Sistema de Información de Mercados Mayoristas, PIMA, Heredia, Costa Rica, 2010.

[6] H. Quesada, Interviewee, Cultivo de tacaco en Grecia, Alajuela, Costa Rica. [Entrevista]. 2015.

[7] C. Gamboa, Interviewee, Cultivo de tacaco en Montes de Oro, Puntarenas, Costa Rica. [Entrevista]. 2015.

[8] O. Varela, Interviewee, Cultivo de tacaco en la península de Nicoya, Costa Rica. [Entrevista]. 2015.

[9] C. Chízmar, Plantas comestibles de Centroamérica, Santo Domingo, Heredia, Costa Rica: Instituto Nacional de Biodiversidad, 2009.

[10] M. d. M. Cerdas-Araya y J. Castro-Chinchilla, "Caracterización poscosecha de frutos de tacaco (Sechium edule) en Cartago, Costa Rica,» Agronomía Mesoamericana, vol. 28, nº 1, pp. 141-148, 2017.

[11] J. Morales, «Morfología general del tacaco, Sechium tacaco (Cucurbitaceae),» Revista de Biología Tropical, vol. 42, n 1-2, pp. 59-71, 1994.

[12] M. Alfaro, «El tacaco,» Universidad de Costa Rica, San José, Costa Rica, 1941.

[13] J. Morales, «Morfología de Sechium tacaco (Pitt.) Jeffrey. Cucurbitaceae,» Universidad de Costa Rica, San José, Costa Rica, 1991.

[14] J. C. Saborío, «Estudio fenológico del tacaco [Sechium tacaco (Pittier) C. Jeffrey], en Santa Lucía, Barva, Heredia,» Universidad Nacional, Heredia, Costa Rica, 1998. 
[15] J. C. Saborío, A. Brenes y S. Munguía, «Comportamiento del rendimiento en una población experimental de tacaco [Sechium tacaco (Pittier) C. Jeffrey], localizada en Barva, Heredia,» de Memoria XI Congreso Nacional Agronómico y de Recursos Naturales, 19-23 julio, San José, Costa Rica, 1999.

[16] J. C. Saborío, A. Brenes y S. Munguía, «Variación de los caracteres morfométricos de frutos y semillas de tacaco [Sechium tacaco (Pittier) C. Jeffrey], a lo largo del ciclo productivo de la planta,» de Memoria XI Congreso Nacional Agronómico y de Recursos Naturales, 19-23 julio, San José, Costa Rica, 1999.

[17] A. Brenes, «Algunas apreciaciones sobre posibles estrategias de manejo y mejoramiento genético del tacaco [Sechium tacaco (Pittier) C. Jeffrey],»Imágenes, vol. 5, n 8, pp. 77-80, 1998.

[18] A. Brenes, «Conservación y utilización racional de germoplasma de Sechium en Costa Rica. Proyecto colaborativo entre diversas entidades,»Imágenes, vol. 3, n 6, pp. 71-78, 1996.

[19] M. A. Sibaja, «Puede desaparecer única especie agrícola autóctona: Costa Rica,» Germinar, n² 2, pp. 3-4, 1991. 